\title{
A Video Guided Solution for Screw Insertion in Orthopedic Plate Fixation
}

\author{
J. Magaraggia ${ }^{a, b}$, G. Kleinszig ${ }^{b}$, R. Graumann ${ }^{b}$, E. Angelopoulou ${ }^{a}$, J. Hornegger ${ }^{a}$ \\ ${ }^{a}$ Pattern Recognition Lab, University of Erlangen-Nuremberg, Erlangen, Germany \\ ${ }^{b}$ Siemens AG, Healthcare Sector, Erlangen, Germany
}

\begin{abstract}
In orthopedic and trauma surgery, metallic plates are used for reduction and fixation of bone fractures. In clinical practice, the intra-operative planning for screw fixation is usually based on fluoroscopic images. Screw fixation is then performed on a free-hand basis. As such, multiple attempts may be required in order to achieve an optimal positioning of the fixing screws. To help the physician insert the screws in accordance to the planned position, we propose a method for screw insertion guidance. Our approach uses a small video camera, rigidly placed on the drill, and a set of small markers that are rigidly fixed on a variable angle drill sleeve. In order to investigate the achievable accuracy of our setup, we simulate the estimation of the drill bit position under two different marker arrangements, planar and 3D, and different noise levels. Furthermore, we motivate our choices for marker design and position given the limited space available for marker positioning, the requirement for accurate position estimation of the drill bit and the illumination changes that could affect the surgical site. We also describe our proposed marker detection and tracking pipeline. Our simulation results let us conclude that we can achieve an accuracy of $1^{\circ}$ and $1 \mathrm{~mm}$ in the estimation of angular orientation and tip position of the drill bit respectively, provided that we have accurate marker detection.
\end{abstract}

Keywords: Video-Guided Method, Marker Detection, Orthopedic and Trauma Surgery, Locking Screw Plates

\section{INTRODUCTION}

In orthopedic and trauma surgery, fracture reduction and fixation involves the placement of a metallic plate that ideally fits the anatomical fragments that need to be realigned and hold in place. The recently introduced variable angle screw plates (e.g. Smith\&Nephew Inc., Synthes Inc. and Stryker Inc.) permit the insertion of screws with up to $15^{\circ}$ of variable orientation w.r.t the surface plate normal. Insertion depth is usually checked using a depth gauge. Inspection of screw orientation is mostly achieved by acquiring fluoroscopic images so that the image plane is parallel to the screw axis. In both cases, positioning control is a post-drilling step. Consequently, subsequent drilling may be required in order to correct for wrong screw positioning. In fact, accurate screw positioning plays a critical role in the success of the surgical procedure and the recovery of good locomotor function.

In the last decades, several solutions have been proposed in order to guide the physician during the procedure. Typically, optical navigation solutions (e.g. VectorVision ${ }^{\circledR}$ from BRAINLAB AG. and Navigation System II from Stryker Inc.) employ an infrared stereo camera placed outside the operative field and a set of markers attached to the instrument to be tracked. Such solutions are often used for providing navigation in several regions of the body, for example in pedicle screw fixation in the spinal column or arthroscopic interventions at the foot's articulation. Such systems require a clear line of sight between the stereo camera and the markers placed on the instrument. Attention needs, therefore, to be paid by the physician when moving in order not to obstruct the line of sight. Methods employing video cameras have also been proposed. Diotte et al. ${ }^{1}$ exploited an augmented C-Arm where a video camera and a set of mirrors, that are attached to the C-Arm source, allow the acquisition of camera images directly registered with the C-Arm projection images. Furthermore, the use of a modified Schanz screw permits to determine the tip position of the drilling guide only exploiting the camera

The presented method is investigational use and is limited to investigational use. It is not commercially available and its future availability cannot be ensured.

Medical Imaging 2013: Image-Guided Procedures, Robotic Interventions, and Modeling, edited by David R. Holmes, Ziv R. Yaniv, Proc. of SPIE Vol. 8671, 86710X (2013) doi:10.1117/12.2007718; http://dx.doi.org/10.1117/12.2007718

Copyright 2013 Society of Photo-Optical Instrumentation Engineers. One print or electronic copy may be made for personal use only. Systematic reproduction and distribution, duplication of any material in this paper for a fee or for commercial purposes, or modification of the content of the paper are prohibited. 
images. Reaungamornrat et al. ${ }^{2}$ proposed the use of the MicronTracker (Claron, Toronto ON). This tracker, based on a stereo video camera, is mounted on the C-Arm. Registration between camera images and X-ray images is achieved by means of a reference marker visible both in the camera and the X-ray images. Both previous solutions assume the use of a C-Arm augmented either with a video camera or a tracker. Worth mentioning is also the solution proposed by ActiViews, Inc. which provides an easy and intuitive method for percutaneous CTguided intervention in the lungs and liver. It is based on a small camera that, by design, can be easily attached to the biopsy needle or an ablation probe. Specially designed markers are then attached to the patient's skin at the site of the needle insertion. The camera-markers system, in combination with a CT scanner, allows one to determine the position of the needle in real time and to visualize it on the acquired CT scans. However, this approach can not be directly transferred to the field of orthopedic and trauma surgery. During a fracture reduction procedure, the site of the fractured bone is exposed. A skin incision is performed and the skin's edges are pulled apart in order to also allow the positioning of the implant. In this context, marker stitching on the skin at the location where a fixating screw has to be placed would not be feasible.

In order to help the physician insert the screws in accordance to the planned position, that is defined based on intra-operative fluoroscopic images, we propose a guidance method for real-time control of insertion depth and orientation of variable angle locking screws. Our method is based on a video camera rigidly mounted on a drill. Markers placed on the surface of a variable angle drill sleeve allow one to determine the position of the camera, and consequently of the drill bit, w.r.t the local coordinate system of the locking screw hole. In the following sections, we investigate the achievable accuracy of our setup and motivate the choice for marker design and position. We also propose an algorithm for marker detection and tracking for a complete system setup.

\section{METHODS}

A schematic representation of our setup is shown in Fig. 1.

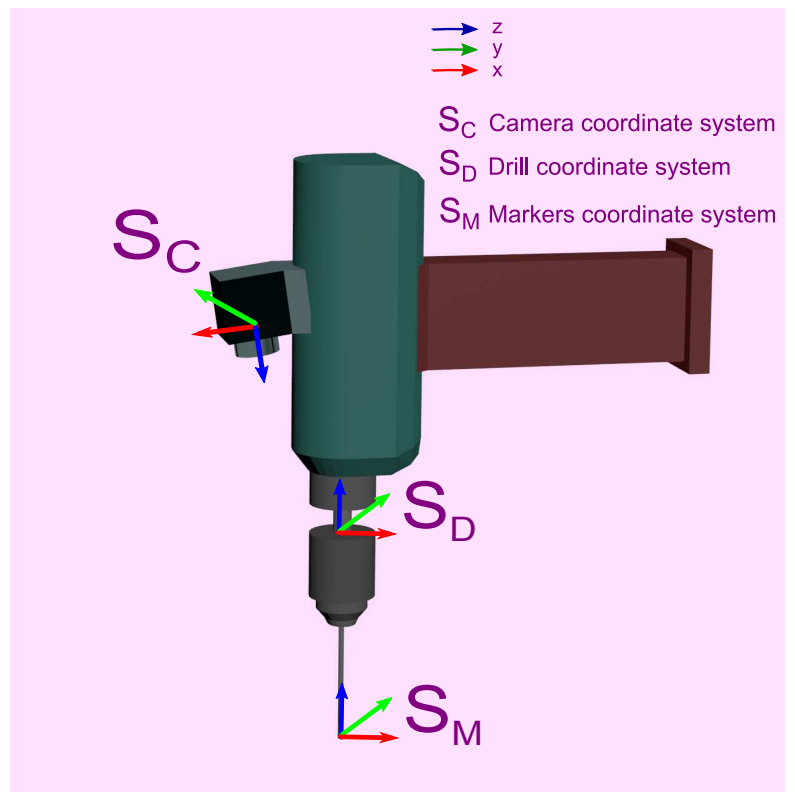

Figure 1. Depiction of our setup and associated coordinate systems

The fixed mounting of a video camera on the surgical instrument will allow us to exploit the camera information in order to determine the position of the instrument itself. Clearly, a reference on the patient is required in order to relate the position of the camera, and thus of the instrument, to the patient. In fracture reductions, drill sleeves are often used in the clinical practice during screw fixation. The usage of a drill sleeve supports

Jessica Magaraggia: jessica.magaraggia@informatik.uni-erlangen.de 
the surgeon in drilling in a predefined direction w.r.t the implant. This direction is usually orthogonal to the plate surface. In the case of variable angle locking plate, screws can usually be oriented up to $\pm 15^{\circ}$ w.r.t the plate normal at the locking hole. When using variable angle locking screw plates, variable angle drill sleeves are employed. The drill sleeve can be placed and removed by the physician on the locking screw hole of interest. We decided to exploit the drill sleeve in order to provide a reference for our instrument position. In particular, markers can be placed onto the surface of the drill sleeve in order to be visible by the camera mounted on the instrument, in our case a surgical drill. When the drill sleeve is positioned in a hole, the origin of the marker coordinate system, $\mathrm{S}_{\mathrm{M}}$, coincides with the center of the locking screw hole. Then, the position of the markers is also known w.r.t the local coordinate system of the hole. As a consequence, the computation of the instrument position w.r.t the coordinate system of the markers placed on the drill sleeve also determines the position of the instrument w.r.t the current locking screw hole on the plate.

Our goal is to simulate the estimation of the drill bit position and orientation given the setup shown in Fig. 1. In real applications, some of the feature points of the markers, that are used for the camera pose estimation, could be occluded or be out of the field of view. A minimum of 4 points is required to recover the camera pose. ${ }^{3}$ We used a set of 8 features points, whose coordinates are known in our world coordinate system $\mathrm{S}_{\mathrm{M}}$ (see Fig. 1). Since the geometry of the system is known, the position of the points in the camera coordinate system $\mathrm{S}_{\mathrm{C}}$ can be directly calculated. Our feature points are thought to be distributed in order to cover a maximum circular region with a diameter of $18 \mathrm{~mm}$ on the drill sleeve. Note that the number of markers and their size need to be reduced as much as possible in order to allow the maximum field of view to the physician. In the first simulation all the points were lying on a plane and the PnP (Perspective-n Points) algorithm by Schweighofer et al. ${ }^{4}$ was used for pose recovery. In the second simulation 1 point was not coplanar with the remaining 8 . In this case, the 3D configuration allowed the use of the PnP algorithm by Lu et al. ${ }^{5}$ For each of the 2 configurations, 4 sets of 800 random poses of the drill were generated. In each of the 4 sets we used a different level of additive Gaussian noise. Noise addition aims to also take into consideration the effect of the drill's intrinsic vibration. The results are summarized in Table 1 and in Table 3.

Let $\mathbf{T}_{\mathbf{M}}^{\mathrm{D}}=\left[\mathbf{R}_{\mathbf{M}}^{\mathrm{D}} \mid \mathbf{t}_{\mathbf{M}}^{\mathrm{D}}\right]$ be the transformation matrix from the coordinate system of the drill $\mathrm{S}_{\mathrm{D}}$ to the markers coordinate system $\mathrm{S}_{\mathrm{M}}$ (see Fig. 1). $\mathbf{R}_{\mathbf{M}}^{\mathrm{D}}$ and $\mathbf{t}_{\mathrm{M}}^{\mathrm{D}}$ are respectively the rotation and translation matrix from $\mathrm{S}_{\mathrm{D}}$ to $\mathrm{S}_{\mathrm{M}}$. Since we are interested in the direction of the drill axis and the depth of insertion of the drill tip, it resulted convenient to describe the position of the origin $\mathbf{O}_{\mathbf{D}}$ of $\mathrm{S}_{\mathrm{D}}$ in terms of a spherical coordinate system. We fixed the parameter $\rho$ to be the length $\mathrm{L}$ of the drill bit. $\theta$ is the angle between the drill bit and the $z$ axis of $\mathrm{S}_{\mathrm{M}}$ and $\phi$ is the angle between the projection of the drill bit onto the $x-y$ plane in $\mathrm{S}_{\mathrm{M}}$ and the positive $x$ axis measured in anti-clock wise direction. Since a variable angle drill sleeve usually allows a value of $\theta \in\left[0^{\circ}, 15^{\circ}\right]$ and $\phi \in\left[0^{\circ}, 360^{\circ}\right]$, these ranges represent also the range of values of the two parameters used in our simulations. Once $\theta, \phi$ and $\rho$ are set, the corresponding tangential axis represent our $S_{\mathrm{D}}$. For each level of noise and for each value of $\theta \in\left[0^{\circ}, 2^{\circ}, \ldots, 14^{\circ}\right], 100$ random values of $\phi$ in the range of $\left[0^{\circ}, 360^{\circ}\right]$ were generated, resulting in 800 random poses for each noise level.

The relative position of the camera w.r.t the drill remained fixed throughout the whole simulation and is described by the transformation matrix $\mathbf{T}_{\mathbf{D}}^{\mathbf{C}}=\left[\mathbf{R}_{\mathbf{D}}^{\mathbf{C}} \mid \mathbf{t}_{\mathbf{D}}^{\mathbf{C}}\right]$. $\mathbf{R}_{\mathbf{D}}^{\mathbf{C}}$ and $\mathbf{t}_{\mathbf{D}}^{\mathbf{C}}$ are respectively the rotation and translation matrix from $S_{C}$ to $S_{D}$.

The final transformation $\mathbf{T}_{\mathbf{C}}^{\mathbf{M}}$ from $\mathrm{S}_{\mathrm{M}}$ to $\mathrm{S}_{\mathrm{C}}$ is described by Eq. 1. Given our set $V=\left\{\mathbf{v}_{\mathbf{i}}\right\}_{i=1, . ., N}$ of key points in $\mathrm{S}_{\mathrm{M}}$, where $\mathbf{v}_{\mathbf{i}}=\left(x_{\mathrm{Mi}}, y_{\mathrm{Mi}}, z_{\mathrm{Mi}}, 1\right)^{T}$, we can directly calculate the corresponding points in $\mathrm{S}_{\mathrm{C}}$ according to Eq. 1 and Eq. 2. We can also calculate the position of our points on the image plane $X=\left\{\mathbf{x}_{\mathbf{i}}\right\}_{i=1, . ., N}$ as described in Eq 3, where $\mathbf{x}_{\mathbf{i}}=\left(x_{i}, y_{i}\right)^{T}$. We assumed our camera to be described by the classical pin-hole model.

$$
\mathbf{T}_{\mathbf{C}}^{\mathrm{M}}=\mathbf{T}_{\mathbf{C}}^{\mathrm{D}} \mathbf{T}_{\mathrm{D}}^{\mathrm{M}}
$$

where

$$
\left[\begin{array}{c}
x_{\mathrm{Ci}} \\
y_{\mathrm{Ci}} \\
z_{\mathrm{Ci}} \\
1
\end{array}\right]=\mathbf{T}_{\mathbf{C}}^{\mathbf{M}}\left[\begin{array}{c}
x_{\mathrm{Mi}} \\
y_{\mathrm{Mi}} \\
z_{\mathrm{Mi}} \\
1
\end{array}\right]
$$




$$
\lambda\left[\begin{array}{c}
x_{i} \\
y_{i} \\
1
\end{array}\right]=\left[\begin{array}{ccc}
f_{\mathrm{x}} & 0 & u_{\mathrm{x}} \\
0 & f_{\mathrm{y}} & u_{\mathrm{y}} \\
0 & 0 & 1
\end{array}\right]\left[\begin{array}{cccc}
1 & 0 & 0 & 0 \\
0 & 1 & 0 & 0 \\
0 & 0 & 1 & 0
\end{array}\right]\left[\begin{array}{c}
x_{\mathrm{Ci}} \\
y_{\mathrm{Ci}} \\
z_{\mathrm{Ci}} \\
1
\end{array}\right]
$$

In Eq. $3, f_{\mathrm{x}}$ and $f_{\mathrm{y}}$ represent the focal length of the camera in terms of pixels, while $u_{\mathrm{x}}$ and $u_{\mathrm{y}}$ represent the principal point.

For each $\mathbf{x}_{\mathbf{i}}$, we calculate the corresponding noisy point $\tilde{\mathbf{x}}_{\mathbf{i}}$ by considering additive Gaussian noise: $\tilde{\mathbf{x}}_{\mathbf{i}}=\mathbf{x}_{\mathbf{i}}+\tilde{\mathbf{n}}$. The components of the noise vector $\tilde{\mathbf{n}}=\left(\tilde{n}_{\mathrm{x}}, \tilde{n}_{\mathrm{y}}\right)^{T}$ follow a Gaussian distribution $\tilde{n}_{\mathrm{i}} \sim \mathcal{N}\left(0, \sigma^{2}\right)$.

As previously mentioned, we first considered pose recovery using 8 coplanar feature points. These 8 feature points can be thought to be the center of 8 circles lying on a planar ring on the surface of the drill sleeve. A representation of such disposition is depicted in Fig. 2a. Such arrangement of the markers would intuitively allow them to be mostly visible in the Field of View (FOV) of the camera and well spread in the camera image. This is the configuration we used in our planar setup. As described by Schweighofer et al., ${ }^{4}$ a planar configuration can lead to ambiguity in the solution of the recovery of the camera pose. In particular, Schweighofer et al. showed that the objective function proposed by $\mathrm{Lu}$ et al. ${ }^{5}$ that is minimized in order to recover the position of the camera, can have two local minima in the case of a planar arrangement of the feature points. They proposed therefore a reformulation of the objective function in order to identify the two local minima and recover both solutions. The pose out of the two that gives the smallest error is considered as the final solution. In our case, since we know that the direction of the axis of the drill can not exceed a deviation of $15^{\circ}$ w.r.t the $\mathrm{z}$ axis of $\mathrm{S}_{\mathrm{M}}$, we can further refine the choice made by the algorithm. We then also check that the final transformation leads to a value of angular orientation $\theta$ smaller than $15^{\circ}$.

In the second arrangement, one of the points is not coplanar. The idea behind this is to take a 3D configuration into consideration and to exploit the prior information concerning the position of the instrument tip before the actual drilling operation starts. Since the position between instrument and camera will be fixed by construction, the position of the instrument tip in the camera image is also unchanged and can be determined at assembly time. No further tip segmentation in the camera images would be required. In the first step of screw fixation, before the actual drilling starts, the drill tip is positioned by the physician at the locking screw hole where the screw needs to be placed. In this pre-drilling phase, the instrument tip position is known w.r.t the marker coordinate system $\mathrm{S}_{\mathrm{M}}$ since it is positioned at the lowest end of the drill sleeve, which is also the origin of our marker coordinate system. The positions of the instrument tip in the image and in $\mathrm{S}_{\mathrm{M}}$ can then be exploited as point correspondence for the pose calculation. However, such correspondence would not hold once the physician starts the drilling.

Once we have $\mathbf{T}_{\mathbf{M}}^{\mathrm{C}}$, we can easily go back to the matrix $\mathbf{T}_{\mathbf{M}}^{\mathrm{D}}$ as reported in Eq. 4 . This can be done since the matrix $\mathbf{T}_{\mathbf{C}}^{\mathrm{D}}$ is fixed by construction. From $\mathbf{T}_{\mathbf{M}}^{\mathrm{D}}$, we are interested in recovering the corresponding values of $\theta$ in order to calculate the corresponding errors. We can calculate $\hat{\theta}$ from the $z$ axis recovered from $\mathbf{T}_{\mathbf{M}}^{\mathrm{D}}, \widehat{\mathbf{z}_{\mathbf{D}}}$, since the $z$ axis of $\mathrm{S}_{\mathrm{D}}$ is oriented along the axis of the drill.

$$
\begin{aligned}
& \mathbf{T}_{\mathbf{M}}^{\mathrm{D}}=\mathbf{T}_{\mathbf{M}}^{\mathbf{C}} \mathbf{T}_{\mathbf{C}}^{\mathrm{D}} \\
& \hat{\theta}=\arccos \widehat{\mathbf{z}_{\mathbf{D}}}
\end{aligned}
$$

In our simulated model, the position of the instrument tip $\mathbf{P}_{\mathbf{D}}=\left(x_{\mathrm{D}}, y_{\mathrm{D}}, z_{\mathrm{D}}, 1\right)^{T}$ in the drill coordinate system $\mathrm{S}_{\mathrm{D}}$ is also known and remains constant since no relative movement occurs between $\mathbf{P}_{\mathbf{D}}$ and $\mathrm{S}_{\mathrm{D}}$. For each randomly generated pose, the position of the tool tip $\mathbf{P}_{\mathbf{M}}=\left(x_{\mathrm{M}}, y_{\mathrm{M}}, z_{\mathrm{M}}, 1\right)^{T}$ w.r.t $\mathrm{S}_{\mathrm{D}}$ is also known. Once we estimate $\mathbf{T}_{\mathbf{M}}^{\mathrm{D}}$ for the current pose, we can exploit it to recover the tool tip position in the marker coordinate system $\widehat{\mathbf{P}_{\mathbf{M}}}$ as follows:

$$
\widehat{\mathbf{P}_{M}}=\mathbf{T}_{M}^{\mathrm{D}} \mathbf{P}_{\mathbf{D}}
$$




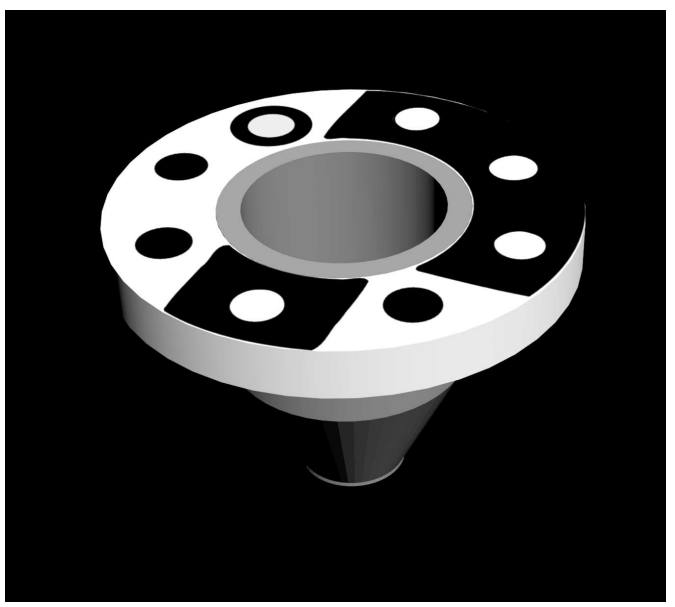

(a) Circular point distribution

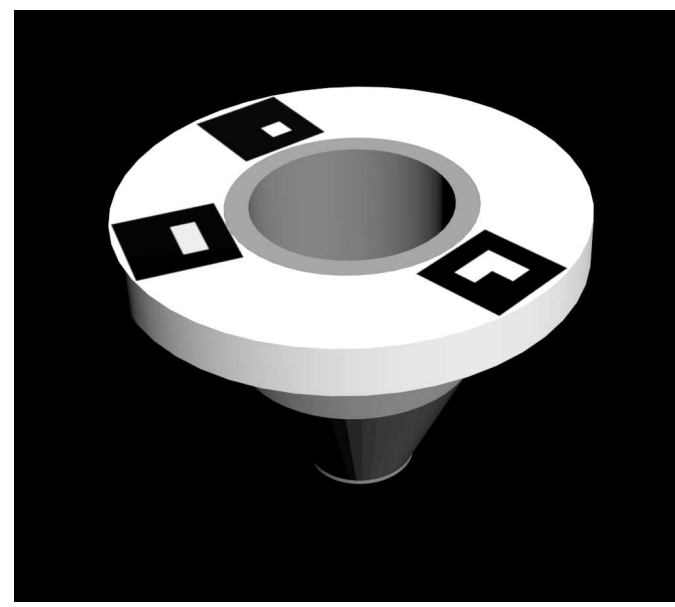

(b) Square point distribution

Figure 2. Depiction of the two point configurations considered.

Around the site of the fixing plate, skin edges are usually kept apart by a surgical retractor in order for the physician to access the fractured bone. However, ligaments and tendons can still be pressuring at the borders of the plate. Consequently, when a drill sleeve is positioned at one screw hole for drilling, it can also be subjected to their interference. Thus, the markers area must be small in order to reduce the interference with the surrounding tissues and not to obstruct the FOV of the surgical site to the doctor. The presence of the markers inevitably increases the total volume occupied by the drill sleeve.

During the simulation, our feature points were considered distributed on a circular surface attached to the drill sleeve. Such a configuration would favor the usage of circular markers, like the ones shown in Fig. 2a, for example. The feature point would be represented by the center of the circle. In this arrangement, the feature points are homogeneously distributed on the circular surface. Circular markers are easy to detect and their size could be easily adapted to spacial constraints. Furthermore, the calculation of the centroid in an the image is regarded as a robust computation. However, there are also some disadvantages. It is known that the center of the circle does not correspond to the center of the ellipse that one can observe in an image due to the effect of perspective projection. ${ }^{6}$ The feature correspondence between the center of the detected ellipse and the known 3D position of the center of the circle has therefore an intrinsic inaccuracy. Moreover, each circular marker provides just one feature point that can be used for the calculation. In augmented reality applications, square markers are often preferred ${ }^{7,8,9}$ because their corners are considered robust features ${ }^{10}$ for identification and tracking. Each square provides 4 feature points that can be used for pose recovery. The employment of square markers results in a different feature point distribution and its impact on the recovery of the drill position needs also to be investigated. We ran therefore another test. The only difference in the setup is the distribution of the feature points, that now are arranged as the 12 corners of the 3 square markers depicted in Fig. $2 \mathrm{~b}$. Our feature points are still distributed on a circular region with a diameter of $18 \mathrm{~mm}$. The results are reported in Table 2 and in Table 4.

In our implementation, we plan to exploit square markers containing a 4-bits binary code as identification. Furthermore, since the operative site is frequently affected by variations in illumination, we chose black and white markers to take advantage of their intrinsic high contrast. Monochrome markers allow us also to naturally employ a monochrome camera, which is known to provide better contrast than a corresponding RGB-camera and does not suffer from chromatic aberrations. Hence, it is expected to provide better accuracy in feature detection. Similar to the markers proposed by Forman et al. ${ }^{9}$ all the bits are used for encoding. No redundant code is present. Moreover, just 3 of the possible 16 configurations are going to be used (see Fig. 2b). In this way, depending on the order in which the internal binary code is read in the image, it is possible to univocally associate with each corner its corresponding $3 \mathrm{D}$ coordinate in $\mathrm{S}_{\mathrm{M}}$. The appearance of the markers on the drill sleeve is depicted in Fig. 2b.

For the detection of the square markers, similar steps to the ones described in $^{8}$ can be employed. The image 


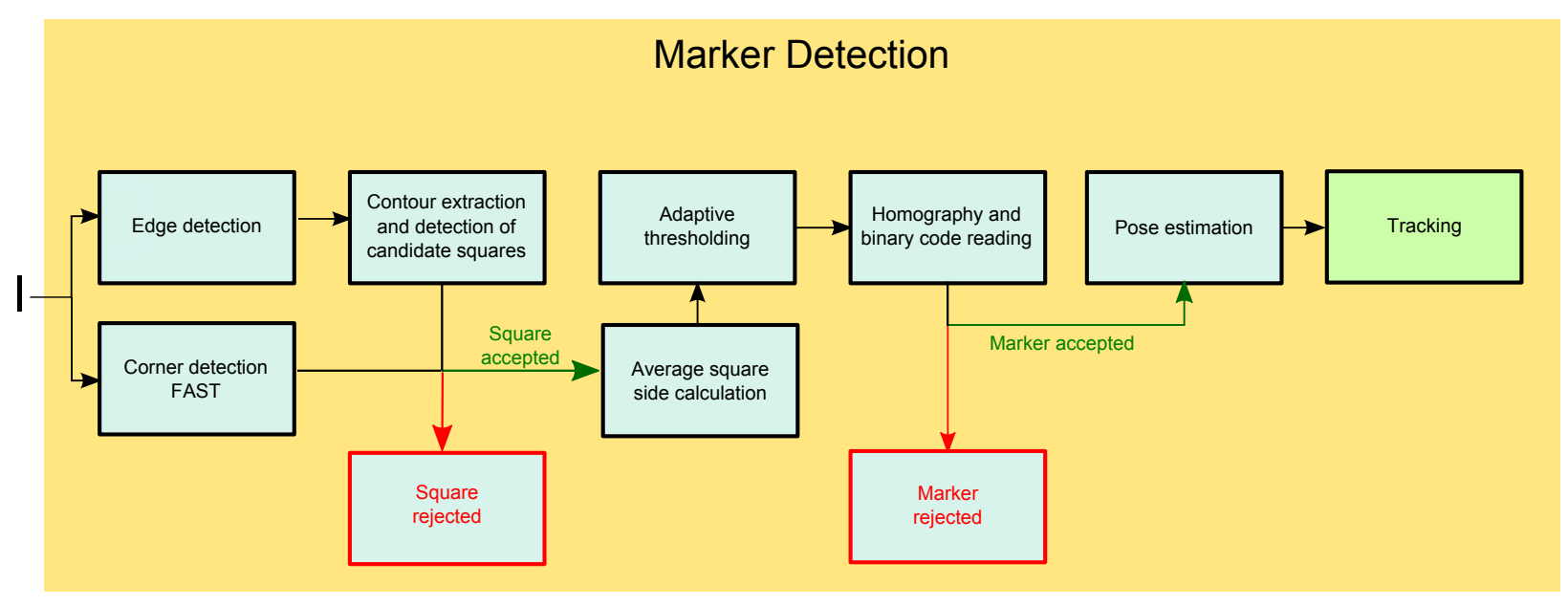

Figure 3. Marker detection block diagram

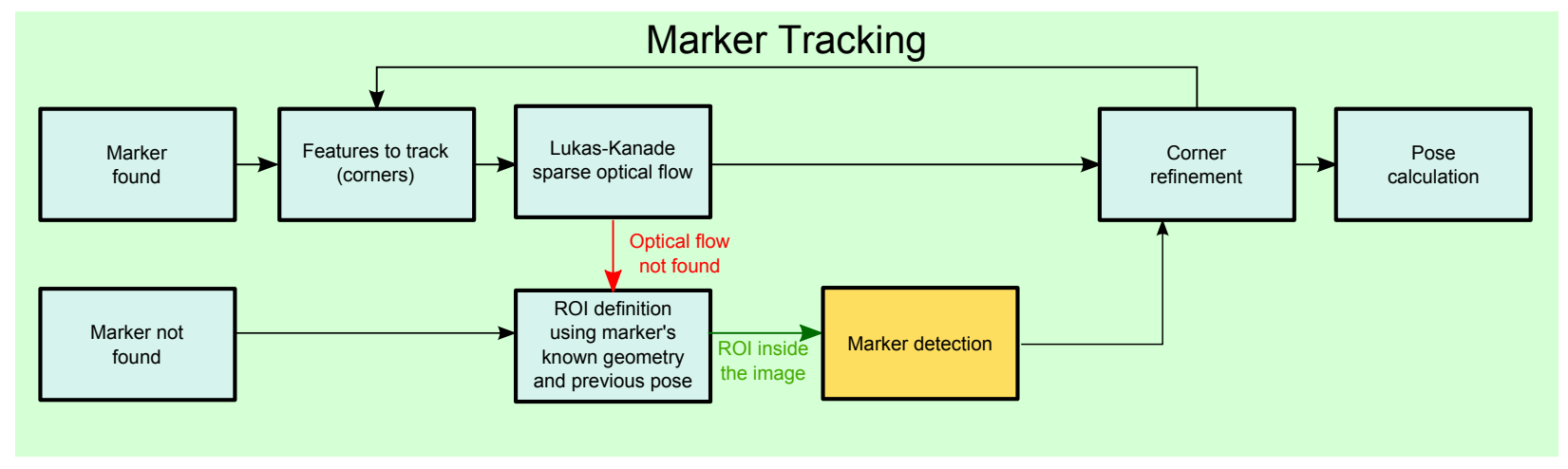

Figure 4. Marker tracking block diagram

in input $\mathrm{I}$ is processed once for edge detection $\left(\mathrm{I}_{\mathrm{E}}\right)$ and once for corner detection using $\mathrm{FAST}^{11}\left(\mathrm{I}_{\mathrm{C}}\right)$. Image contours are then approximated by a polygonal curve. Polygons with 4 sides are considered candidates. If for at least 3 vertices of the polygon, a corner was detected inside a $5 \times 5$ window centered on the polygon vertex, the candidate is further processed. The previous step is needed since the vertices of the polygon may not correspond to real corners in the image. The average length of all polygons sides is then used as a window kernel size to perform adaptive thresholding on I. The vertices of the candidates are used to perform a homography and read the internal binary code. If the binary code coincides with one of the employed codes, a marker was found. Corners are refined using the cvFindCornerSubPix function of the OpenCV library. ${ }^{12}$ Once at least four 2D3D correspondences are established, it is possible to calculate the camera pose. The steps of the algorithm are summarized in Fig. 3. After a marker is detected, its corners are used as features to be tracked. For our purposes, features are tracked via the sparse optical flow implementation by Bouget of the Lukas-Kanade algorithm. ${ }^{13}$ While tracking, a marker can be lost because the flow for its corners could not be found in the current frame. The latter can result from sudden illumination changes which could be caused for example by the physician while moving or re-positioning a surgical light. If a marker was lost, the camera pose calculated for the previous frame from the visible markers is used to project the 3D coordinates of the missing marker onto the image plane. If the ROI so defined is inside the image, it is processed with the previously defined marker detection algorithm. After corner refinement, the camera pose is calculated exploiting all sets of available 2D-3D correspondences. The steps of the tracking pipeline are summarized in Fig. 4. 


\section{RESULTS}

The results of the simulations exploiting a circular distribution of the feature points are summarized in Tables 1 and 3. The results of the second test concerning a distribution of the feature points belonging to square markers are reported in Tables 2 and 4. The rotation and translation errors were calculated according to Eq. 7 and Eq 8 respectively. The errors concerning the position of the drill bit were calculated according to Eq. 9. The error $e_{\mathrm{IT}}$ is the error in the estimation of the instrument tip position. Since the origin of $\mathrm{S}_{\mathrm{M}}$ also represents the point at which the drilling starts, $e_{\mathrm{IT}}$ expresses also the error in the insertion depth.

$$
\begin{gathered}
e_{\mathrm{R}}=\arccos \frac{\operatorname{trace}\left(\widehat{\mathbf{R}_{\mathbf{M}}^{\mathrm{D}}}{\overline{\mathbf{R}_{\mathbf{M}}^{\mathrm{D}}}}^{\mathbf{T}}\right)-\mathbf{1}}{2} \\
e_{\mathrm{T}}=\left\|\widehat{\mathbf{t}_{\mathbf{M}}^{\mathrm{D}}}-\overline{\mathbf{t}_{\mathbf{M}}^{\mathrm{D}}}\right\|_{\mathbf{2}}
\end{gathered}
$$

In Eq. 7 and $8 \widehat{\mathbf{R}_{\mathbf{M}}^{\mathrm{D}}}$ and $\widehat{\mathbf{t}_{\mathbf{M}}^{\widehat{\mathrm{P}}}}$ are the estimated rotation and translation components of the transformation matrix $\mathbf{T}_{\mathbf{M}}^{\mathrm{D}}$, while $\overline{\mathbf{R}_{\mathrm{M}}^{\mathrm{D}}}$ and $\overline{\mathbf{t}_{\mathrm{M}}^{\mathrm{D}}}$ are the corresponding true values.

$$
e_{\theta}=|\widehat{\theta}-\bar{\theta}| \quad e_{\mathrm{IT}}=\left\|\widehat{\mathbf{P}_{\mathbf{M}}}-\overline{\mathbf{P}_{\mathbf{M}}}\right\|_{\mathbf{2}}
$$

In Eq. $9 \widehat{\theta}$ and $\widehat{\mathbf{P}_{\mathbf{M}}}$ are the estimated axis orientation and tip position, while $\bar{\theta}$ and $\overline{\mathbf{P}_{\mathbf{M}}}$ are the corresponding true values.

Table 1. Results from the Schweighofer's algorithm. ${ }^{4} \sigma^{2}$ is the variance of the Gaussian noise. Shown are errors in: the estimation of the rotation matrix of $\mathrm{S}_{\mathrm{D}}$ w.r.t $\mathrm{S}_{\mathrm{M}}, e_{\mathrm{R}}$, the translation of $\mathrm{S}_{\mathrm{D}}$ w.r.t $\mathrm{S}_{\mathrm{M}}, e_{\mathrm{T}}$, the orientation of the drill axis, $e_{\theta}$, and the error in the tip position, $e_{\mathrm{IT}}$.

\begin{tabular}{l|c|c|c|c}
\hline \multicolumn{5}{c}{ Circular Configuration: Planar Arrangement of the Feature Points } \\
\hline$\sigma^{2}(\mathrm{px})$ & $e_{\mathrm{R}}\left({ }^{\circ}\right)$ & $e_{\mathrm{T}}(\mathrm{mm})$ & $e_{\theta}\left({ }^{\circ}\right)$ & $e_{\mathrm{IT}}(\mathrm{mm})$ \\
\hline 0.5 & $0.52 \pm 0.27$ & $0.31 \pm 0.21$ & $0.32 \pm 0.25$ & $0.26 \pm 0.20$ \\
\hline 1 & $1.01 \pm 0.52$ & $0.62 \pm 0.42$ & $0.59 \pm 0.48$ & $0.53 \pm 0.39$ \\
\hline 1.5 & $1.57 \pm 0.81$ & $0.96 \pm 0.64$ & $0.92 \pm 0.74$ & $0.81 \pm 0.61$ \\
\hline 2 & $2.06 \pm 1.07$ & $1.27 \pm 0.47$ & $1.22 \pm 0.96$ & $1.08 \pm 0.80$ \\
\hline
\end{tabular}

Table 2. Results from the Schweighofer's algorithm. ${ }^{4} \sigma^{2}$ is the variance of the Gaussian noise. Shown are errors in: the estimation of the rotation matrix of $\mathrm{S}_{\mathrm{D}}$ w.r.t $\mathrm{S}_{\mathrm{M}}, e_{\mathrm{R}}$, the translation of $\mathrm{S}_{\mathrm{D}}$ w.r.t $\mathrm{S}_{\mathrm{M}}, e_{\mathrm{T}}$, the orientation of the drill axis, $e_{\theta}$, and the error in the tip position, $e_{\mathrm{IT}}$.

\begin{tabular}{l|c|c|c|c}
\hline \multicolumn{5}{c}{ Square Configuration: Planar Arrangement of the Feature Points } \\
\hline$\sigma^{2}(\mathrm{px})$ & $e_{\mathrm{R}}\left({ }^{\circ}\right)$ & $e_{\mathrm{T}}(\mathrm{mm})$ & $e_{\theta}\left(^{\circ}\right)$ & $e_{\mathrm{IT}}(\mathrm{mm})$ \\
\hline 0.5 & $0.58 \pm 0.32$ & $0.35 \pm 0.25$ & $0.33 \pm 0.28$ & $0.30 \pm 0.24$ \\
\hline 1 & $1.15 \pm 0.63$ & $0.63 \pm 0.49$ & $0.68 \pm 0.56$ & $0.53 \pm 0.46$ \\
\hline 1.5 & $1.74 \pm 0.95$ & $1.03 \pm 0.75$ & $1.03 \pm 0.84$ & $0.87 \pm 0.71$ \\
\hline 2 & $2.29 \pm 1.28$ & $1.40 \pm 1.02$ & $1.35 \pm 1.12$ & $1.18 \pm 0.97$ \\
\hline
\end{tabular}


Table 3. Results from the Lu's algorithm. ${ }^{5} \sigma^{2}$ is the variance of the Gaussian noise. Shown are errors in: the estimation of the rotation matrix of $\mathrm{S}_{\mathrm{D}}$ w.r.t $\mathrm{S}_{\mathrm{M}}, e_{\mathrm{R}}$, the translation of $\mathrm{S}_{\mathrm{D}}$ w.r.t $\mathrm{S}_{\mathrm{M}}, e_{\mathrm{T}}$, the orientation of the drill axis, $e_{\theta}$, and the error in the tip position, $e_{\mathrm{IT}}$.

\begin{tabular}{l|c|c|c|c}
\hline \multicolumn{5}{c}{ Circular Configuration: 3D Arrangement of the Feature Points } \\
\hline$\sigma^{2}(\mathrm{px})$ & $e_{\mathrm{R}}\left({ }^{\circ}\right)$ & $e_{\mathrm{T}}(\mathrm{mm})$ & $e_{\theta}\left(^{\circ}\right)$ & $e_{\mathrm{IT}}(\mathrm{mm})$ \\
\hline 0.5 & $0.29 \pm 0.14$ & $0.22 \pm 0.16$ & $0.17 \pm 0.13$ & $0.20 \pm 0.16$ \\
\hline 1 & $0.58 \pm 0.28$ & $0.46 \pm 0.33$ & $0.34 \pm 0.26$ & $0.41 \pm 0.31$ \\
\hline 1.5 & $0.88 \pm 0.43$ & $0.71 \pm 0.52$ & $0.49 \pm 0.38$ & $0.64 \pm 0.50$ \\
\hline 2 & $1.16 \pm 0.56$ & $0.95 \pm 0.69$ & $0.66 \pm 0.50$ & $0.86 \pm 0.67$ \\
\hline
\end{tabular}

Table 4. Results from the Lu's algorithm. ${ }^{5} \sigma^{2}$ is the variance of the Gaussian noise. Shown are errors in: the estimation of the rotation matrix of $\mathrm{S}_{\mathrm{D}}$ w.r.t $\mathrm{S}_{\mathrm{M}}, e_{\mathrm{R}}$, the translation of $\mathrm{S}_{\mathrm{D}}$ w.r.t $\mathrm{S}_{\mathrm{M}}, e_{\mathrm{T}}$, the orientation of the drill axis, $e_{\theta}$, and the error in the tip position, $e_{\mathrm{IT}}$.

\begin{tabular}{l|c|c|c|c}
\hline \multicolumn{5}{c}{ Square Configuration: 3D Arrangement of the Feature Points } \\
\hline$\sigma^{2}(\mathrm{px})$ & $e_{\mathrm{R}}\left(^{\circ}\right)$ & $e_{\mathrm{T}}(\mathrm{mm})$ & $e_{\theta}\left(^{\circ}\right)$ & $e_{\mathrm{IT}}(\mathrm{mm})$ \\
\hline 0.5 & $0.29 \pm 0.14$ & $0.21 \pm 0.15$ & $0.17 \pm 0.13$ & $0.19 \pm 0.15$ \\
\hline 1 & $0.58 \pm 0.29$ & $0.45 \pm 0.36$ & $0.33 \pm 0.26$ & $0.40 \pm 0.31$ \\
\hline 1.5 & $0.86 \pm 0.43$ & $0.68 \pm 0.49$ & $0.50 \pm 0.39$ & $0.61 \pm 0.47$ \\
\hline 2 & $1.14 \pm 0.57$ & $0.86 \pm 0.64$ & $0.65 \pm 0.50$ & $0.77 \pm 0.62$ \\
\hline
\end{tabular}

\section{DISCUSSION AND CONCLUSIONS}

We propose a guidance method for real-time control of the tip position and orientation of variable angle locking screws. Our method aims to allow drilling guidance while introducing minimal additional instrumentation into the operating field. No fluoroscopic images would be required for monitoring. Moreover, if the implant would be registered to intra-operative fluoroscopic images, our method could directly provide drill bit position w.r.t the patient anatomy. In classical navigation systems (e.g. VectorVision ${ }^{\circledR}$ from BRAINLAB Inc. and Navigation System II from Stryker Inc.), the physician needs to pay attention when moving, so that he does not obstruct the line of sight between the optical markers on the instrument and the stereo camera placed outside the operative field. In comparison, our method could allow more flexibility, since such caution would not be required any more. Our simulations showed that in our simple monocular setup, both a circular feature points distribution and a distribution of feature points belonging to square markers, would allow to achieve an accuracy of $1^{\circ}$ and $1 \mathrm{~mm}$ in the estimation of angular orientation and tip position respectively, provided that we have accurate feature detection. Similar results were obtained with both a circular and a square-like distribution of the feature points. However, we noticed also that a circular feature point distribution lead to a slightly better performance in planar configurations. Such a difference was not observed in the case of a 3D configuration. From our tests, we can conclude that both a circular and a square-like arrangement could be employed. However, it is also clear from the results, that the achievable accuracy depends on the noise in the feature points. As discussed previously in this paper, square markers are often preferred because their corners can be detected in a robust way. ${ }^{10}$ For this reason, we suggest an implementation of the system that exploits square markers.

Our analysis also showed that a 3D distribution of the feature points leads to a better accuracy and it is more robust to the noise level. This suggests that a different arrangement of the markers on the drill sleeve is to be preferred. We currently assume that the markers are located on a planar surface that could be attached to the drill sleeve. A 3D arrangement of the feature points could therefore be exploited just in the first step of the surgical procedure, before the drilling starts. As previously mentioned, the information concerning the tip of the drill bit can also be exploited. In order to be able to take advantage of a 3D distribution of the feature points, an alternative positioning of the markers on the drill sleeve is to be preferred. The employment of 
square markers would also be advantageous, since locating a single marker to another position on the drill sleeve, implies a different arrangement of 4 feature points simultaneously. It would facilitate therefore the creation of a $3 \mathrm{D}$ configuration in our setup. As part of our future work, we plan to perform an evaluation of our complete setup exploiting a 3D configuration of the square markers and our proposed pipeline for marker detection and tracking.

\section{REFERENCES}

[1] Diotte, B., Fallavollita, P., Wang, L., Weidert, S., Thaller, P., Euler, E., and Navab, N., "Radiation-free drill guidance in interlocking of intramedullary nails," Medical Image Computing and Computer-Assisted Intervention-MICCAI , 18-25 (2012).

[2] Reaungamornrat, S., Otake, Y., Uneri, A., Schafer, S., Mirota, D., Nithiananthan, S., Stayman, J., Khanna, A., Reh, D., Gallia, G., et al., "Tracker-on-C for cone-beam CT-guided surgery: evaluation of geometric accuracy and clinical applications," Proc. SPIE Medical Imaging 2012: Image-Guided Procedures, Robotic Interventions, and Modeling 8316 (2012).

[3] Ma, Y., [An invitation to 3-d vision: from images to geometric models], vol. 26, Springer Verlag (2004).

[4] Schweighofer, G. and Pinz, A., "Robust pose estimation from a planar target," IEEE Transactions on Pattern Analysis and Machine Intelligence 28(12), 2024-2030 (2006).

[5] Lu, C., Hager, G., and Mjolsness, E., "Fast and globally convergent pose estimation from video images," IEEE Transactions on Pattern Analysis and Machine Intelligence 22(6), 610-622 (2000).

[6] Heikkila, J., "Geometric camera calibration using circular control points," IEEE Transactions on Pattern Analysis and Machine Intelligence 22(10), 1066-1077 (2000).

[7] Fiala, M., "ARTag, a fiducial marker system using digital techniques," Proc. Computer Vision and Pattern Recognition 2, 590-596 (2005).

[8] Wagner, D., Langlotz, T., and Schmalstieg, D., "Robust and unobtrusive marker tracking on mobile phones," ISMAR, International Symposium on Mixed and Augmented Reality, 121-124 (2008).

[9] Forman, C., Aksoy, M., Hornegger, J., and Bammer, R., "Self-encoded marker for optical prospective head motion correction in MRI," Medical Image Computing and Computer-Assisted Intervention-MICCAI, 259266 (2010).

[10] Shi, J. and Tomasi, C., "Good features to track," Proc. Computer Vision and Pattern Recognition-CVPR , 593-600 (1994).

[11] Rosten, E. and Drummond, T., "Machine learning for high-speed corner detection," Proc. European Conference on Computer Vision-ECCV , 430-443 (2006).

[12] Intel Corporation, M. R. L., "OpenCV: Open source computer vision library," (2012).

[13] Bouguet, J., "Pyramidal implementation of the affine Lucas Kanade feature tracker description of the algorithm," Intel Corporation (2001). 